\title{
Microsurgical clipping of ophthalmic artery aneurysms: surgical results and visual outcomes with 208 aneurysms
}

\author{
Tomoya Kamide, MD, ${ }^{1}$ Halima Tabani, MD, ${ }^{1}$ Michael M. Safaee, MD, ${ }^{1}$ Jan-Karl Burkhardt, MD, ${ }^{1}$ and \\ Michael T. Lawton, MD1,2
}

1Department of Neurological Surgery, University of California, San Francisco, California; and 'Department of Neurological Surgery, Barrow Neurological Institute, St. Joseph's Hospital \& Medical Center, Phoenix, Arizona

\begin{abstract}
OBJECTIVE While most paraclinoid aneurysms can be clipped with excellent results, new postoperative visual deficits are a concern. New technology, including flow diverters, has increased the popularity of endovascular therapy. However, endovascular treatment of paraclinoid aneurysms is not without procedural risks, is associated with higher rates of incomplete aneurysm occlusion and recurrence, and may not address optic nerve compression symptoms that surgical debulking can. The increasing endovascular management of paraclinoid aneurysms should be justified by comparisons to surgical benchmarks. The authors, therefore, undertook this study to define patient, visual, and aneurysm outcomes in the most common type of paraclinoid aneurysm: ophthalmic artery (OphA) aneurysms.
\end{abstract}

METHODS Results from microsurgical clipping of 208 OphA aneurysms in 198 patients were retrospectively reviewed. Patient demographics, aneurysm morphology (size, calcification, etc.), clinical characteristics, and patient outcomes were recorded and analyzed.

RESULTS Despite $20 \%$ of these aneurysms being large or giant in size, complete aneurysm occlusion was accomplished in $91 \%$ of 208 cases, with OphA patency preserved in $99.5 \%$. The aneurysm recurrence rate was $3.1 \%$ and the retreatment rate was $0 \%$. Good outcomes (modified Rankin Scale score $0-2$ ) were observed in $96.2 \%$ of patients overall and in all 156 patients with unruptured aneurysms. New visual field defects (hemianopsia or quadrantanopsia) were observed in 8 patients $(3.8 \%)$, decreased visual acuity in $5(2.4 \%)$, and monocular blindness in $9(4.3 \%)$. Vision improved in $9(52.9 \%)$ of the 17 patients with preoperative visual deficits.

CONCLUSIONS The most important risk associated with clipping OphA aneurysms is a new visual deficit. Meticulous microsurgical technique is necessary during anterior clinoidectomy, aneurysm dissection, and clip application to optimize visual outcomes, and aggressive medical management postoperatively might potentially decrease the incidence of delayed visual deficits. As the results of endovascular therapy and specifically flow diverters become known, they warrant comparison with these surgical benchmarks to determine best practices.

https://thejns.org/doi/abs/10.3171/2017.7.JNS17673

KEY WORDS ophthalmic artery aneurysm; microsurgical clipping; visual deficit; hemianopsia; blindness; anterior clinoidectomy; vascular disorders

$\mathrm{C}$ EREBRAL aneurysms arising from the internal carotid artery (ICA) between the cavernous sinus exit (proximal dural ring) and the posterior communicating artery (PCoA) constitute paraclinoid aneurysms. ${ }^{4,10}$ Direct surgery for paraclinoid aneurysms remains a technical challenge because they occupy a tiny region packed with critical structures that include the $\mathrm{C} 5$ and C6 segments of ICA, their branches and perforating arteries, and the optic apparatus, cavernous sinus, and oculomotor nerve. ${ }^{4-6,13,17}$ As if the anatomical complexity here were not enough, these structures are concealed by dura and bone that must be incised and drilled, respectively, to even see them. Anterior clinoidectomy, optic strut removal, and distal dural ring dissection are essential to the exposure and safe clipping of paraclinoid aneurysms, and technical errors in their execution can cause visual deficits,

ABBREVIATIONS ICA = internal carotid artery; ICG = indocyanine green; $\mathrm{mRS}=$ modified Rankin Scale; OphA = ophthalmic artery; PCoA = posterior communicating artery; SAH = subarachnoid hemorrhage; UCSF = University of California, San Francisco.

SUBMITTED March 16, 2017. ACCEPTED July 14, 2017.

INCLUDE WHEN CITING Published online January 26, 2018; DOI: 10.3171/2017.7.JNS17673. 
catastrophic bleeding, and strokes. An understanding of this region comes from study with expert neurosurgeons, cadaveric dissection, reviewing operative videos, and personal experience. There is a learning curve that must be overcome, and when it is, the vast majority of paraclinoid aneurysms can be clipped surgically with excellent results. However, advances in endovascular technology, such as the introduction of flow diverters, and increases in their clinical application have eroded the case volume needed to gain microsurgical competency with paraclinoid aneurysms. Inexperienced neurosurgeons may never acquire the necessary skills, and experienced neurosurgeons may not have occasion to use them.

Therefore, paraclinoid aneurysms and their management provoke more controversy than most other aneurysms. Endovascular treatment of paraclinoid aneurysms is associated with a lower rate of complete occlusion and more frequent recurrence compared with microsurgical treatment. ${ }^{6}$ In addition, endovascular treatment may not address the symptoms associated with optic nerve compression that aneurysm deflation or surgical debulking can. Results with flow diverters have been impressive in this region, and patients are attracted to this minimally invasive approach, but complications are observed in $1.4 \%-7.6 \%$ of cases, and the mortality rates are as high

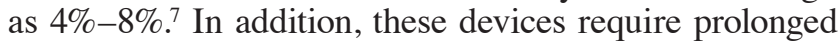
treatment with potent antiplatelet drugs that limit their use in the setting of acute aneurysm rupture and may cause bleeding complications or side effects. Thus, direct surgery for paraclinoid aneurysms remains an indispensable option..$^{21}$ Previous studies demonstrate that microsurgery offers definitive and durable treatment for paraclinoid aneurysms with an acceptable complication rate, 2,10,12,20,21, 23,27 but recent studies document a concerning rate of new visual deficits. ${ }^{15,18,19,22,24,25}$ In addition, the frequency of aneurysm remnants is higher in this region due to limitations in visualizing pathology on the medial wall of the ICA through the narrow corridor of the optic-carotid triangle.

We aimed to better define the results of surgical treatment of the most common paraclinoid aneurysms: ophthalmic artery (OphA) aneurysms. If these aneurysms are increasingly being managed endovascularly, this practice should be justified by comparison with surgical benchmarks. Therefore, we retrospectively reviewed a large microsurgical experience with OphA aneurysms to determine aneurysm and patient outcomes, with particular attention to treatment efficacy and visual complications.

\section{Methods \\ Data Collection}

This study was approved by the University of California, San Francisco (UCSF) institutional review board and conducted in compliance with Health Insurance Portability and Accountability Act regulations. The prospectively maintained database for the vascular neurosurgery service at UCSF was searched for patients with OphA aneurysms who had microsurgical treatment during the 19-year period from December 1997 to July 2016. Patient data were collected from clinical assessments (at admission, during the immediate postoperative period, and at follow-up), ra- diographic films, operative notes, intraoperative imaging, and postoperative angiography. The senior author (M.T.L.) performed microsurgical treatment and reported all operative notes. OphA aneurysms were defined as aneurysms arising in clear relation to the OphA and distinguished strictly from other paraclinoid aneurysms (i.e., superior hypophyseal artery, clinoidal ICA, carotid cave, ventral carotid wall, and dorsal carotid wall aneurysms) during the surgical procedure. Patients treated with bypass or without aneurysm neck clipping (aneurysms treated with wrapping or unclippable) were excluded from the study, as were those with incomplete data.

\section{Microsurgical Technique}

The patient's neck was included in the sterile operative field for compression of the cervical ICA, and we exposed the cervical ICA before craniotomy if retrograde suction decompression was planned or proximal control of the cervical ICA was thought to be necessary. A standard pterional craniotomy was performed with wide sylvian fissure splitting. Intradural clinoidectomy was performed routinely. The distal dural ring was incised circumferentially around the ICA and into the carotid cave medially (Figs. 1-3). A simple straight or curved clip was used for small, narrow-necked aneurysms (Fig. 1 and 2), and a side-angled clip was used for many larger aneurysms because its blades parallel the ophthalmic segment of the ICA. Broad-based and/or giant aneurysms were clipped using "tandem clipping," with a fenestrated clip encircling the lateral neck and closing the medial neck, and a stacked straight clip closing the fenestration and the lateral neck (Fig. 1). OphA aneurysm remnants can occur medially below the tips of the tandem fenestrated clip and laterally within its fenestration. The former is addressed with another overlapping fenestrated clip with a short blade that closes the remnant and transmits the blades of the other clips through its fenestration. The latter is addressed with curved clips within the fenestration of the first fenestrated clip. When associated with another ipsilateral aneurysm, especially one that had ruptured, contralateral OphA aneurysms were clipped when small or medium in size (approximately $<10 \mathrm{~mm}$ ) and judged to be safe for clipping. The contralateral OphA aneurysm was identified underneath the optic nerve through the interoptic triangle. Medially and superiorly projecting aneurysms were easily visualized and clipped without anterior clinoidectomy or dural ring dissection. Permanent clipping with a single curved clip was usually feasible and safe. Aneurysm obliteration and patency of the ICA and branches were confirmed by performing microvascular Doppler ultrasonography and/ or intraoperative catheter angiography during the first 8 years of this series (1997-2005), and intraoperative indocyanine green (ICG) video angiography was performed routinely after 2006 .

\section{Outcome Evaluation}

The following data were collected: age, sex, preoperative visual deficit, aneurysm side and size, number of used clips, type of clip, postoperative neurological visual deficit, and clinical outcome. Aneurysm size was classified 

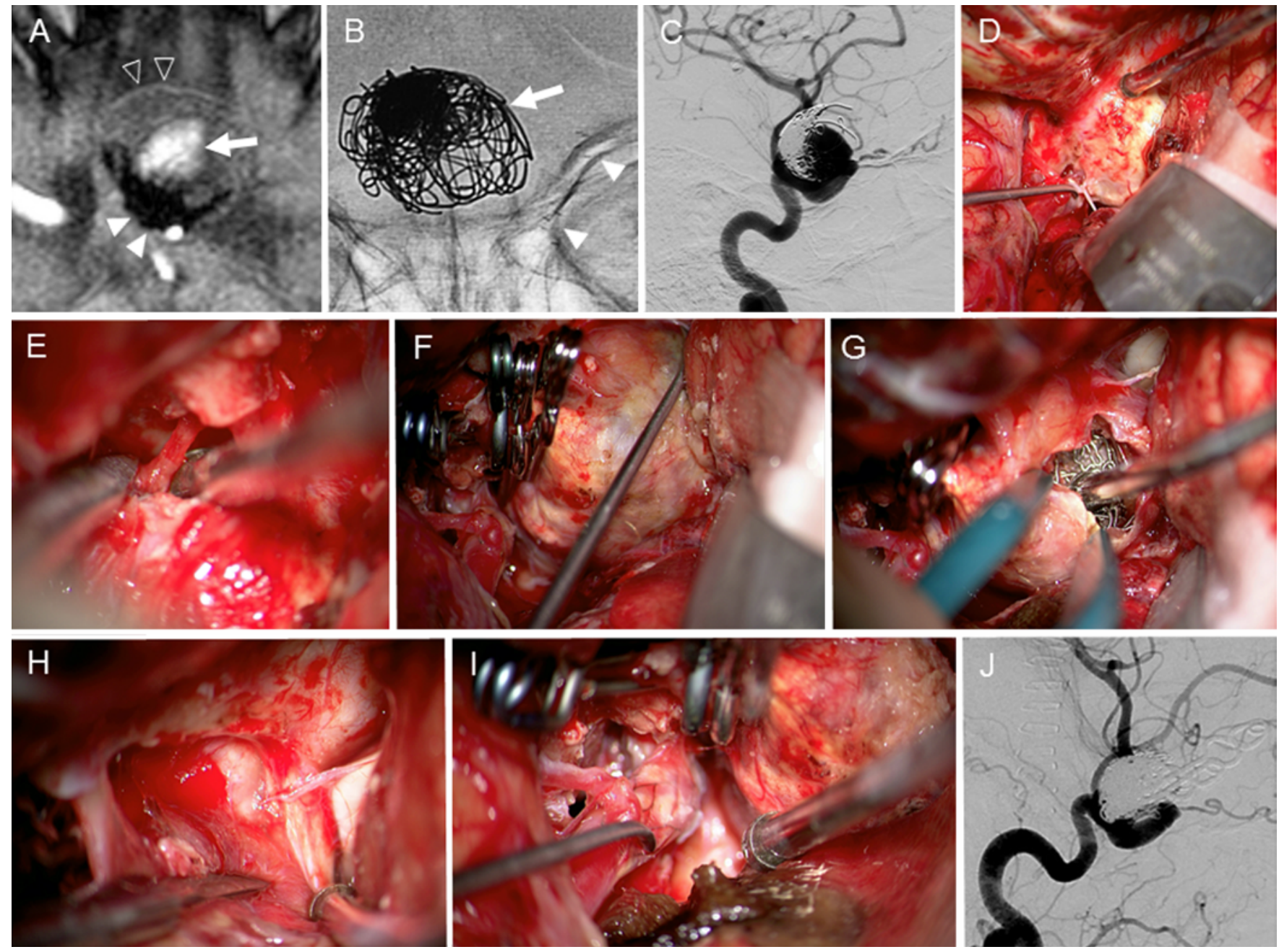

FIG. 1. Illustrative case 1. This 82-year-old woman presented with an unruptured left OphA aneurysm, which was surgically explored and wrapped with cotton 17 years prior. Her aneurysm continued to grow and was treated with stent and coil placement 8 years prior. She progressively lost vision in her left eye to the point of blindness, and when she presented with worsening of visual acuity in the right eye, she was referred for surgery (mRS score 2). MR angiography demonstrated a giant (maximum diameter $25.2 \mathrm{~mm}$ ), thrombotic left OphA aneurysm compressing both optic nerves (A; residual flow [arrow], intraaneurysmal coil mass [solid arrowheads], and intraaneurysmal thrombus [empty arrowheads]). A skull radiograph (anteroposterior view) revealed coil compaction (B; compaction of inserted coils [arrow] and stent markers [solid arrowheads]), and catheter angiography (C; left carotid injection, lateral view) demonstrated aneurysm recurrence. She decided to proceed with microsurgical treatment to prevent further worsening of visual acuity in her right eye. She underwent a left pterional craniotomy with cervical ICA exposure and anterior clinoidectomy. The aneurysm was exposed after splitting of the sylvian fissure (D). Extensive dissection of the distal dural ring exposed the OphA origin (E), and the neck was closed with tandem clipping, using 2 stacked fenestrated clips to encircle the calcified neck proximally and a stacked straight clip to close the fenestration (F). After that, the aneurysm was opened and thrombectomy was performed with ultrasonic aspiration down to the coils $(\mathbf{G})$. The debulked aneurysm was dissected away from the right optic nerve to relieve its mass effect $(\mathbf{H})$, and the stent was inspected in the left ICA. Postoperative catheter angiography revealed no residual filling of aneurysm (I). The patient had no further worsening of visual acuity in the right eye, although her left eye remained blind (mRS score 2). This case demonstrates an initial failure of attempted surgical clipping, aneurysm recurrence after stent-assisted coiling, and finally successful clip reconstruction with optic nerve decompression.

as regular $(<12 \mathrm{~mm})$, large $(12-24 \mathrm{~mm})$, and giant $(>25$ $\mathrm{mm})$. Attention was paid to aneurysm morphology, wall calcification, and thrombosis. Aneurysm outcomes were evaluated with postoperative catheter angiography. Patient outcomes were evaluated 6 weeks after hospital discharge and during the last office visit with the modified Rankin Scale (mRS) and divided into good (mRS score 0-2) and poor (mRS score 3-6). Detailed preoperative and postoperative visual examinations were performed in all cases by an independent neurologist and the neurosurgeon performing the procedure, who evaluated the visual fields and visual acuity. In addition, patients who had visual complaints were referred to the neuro-ophthalmologist at our institute for further evaluation. Visual deficits included worsening visual acuity and visual field deficits.

\section{Statistical Analysis}

Quantitative variables were expressed as the mean \pm 

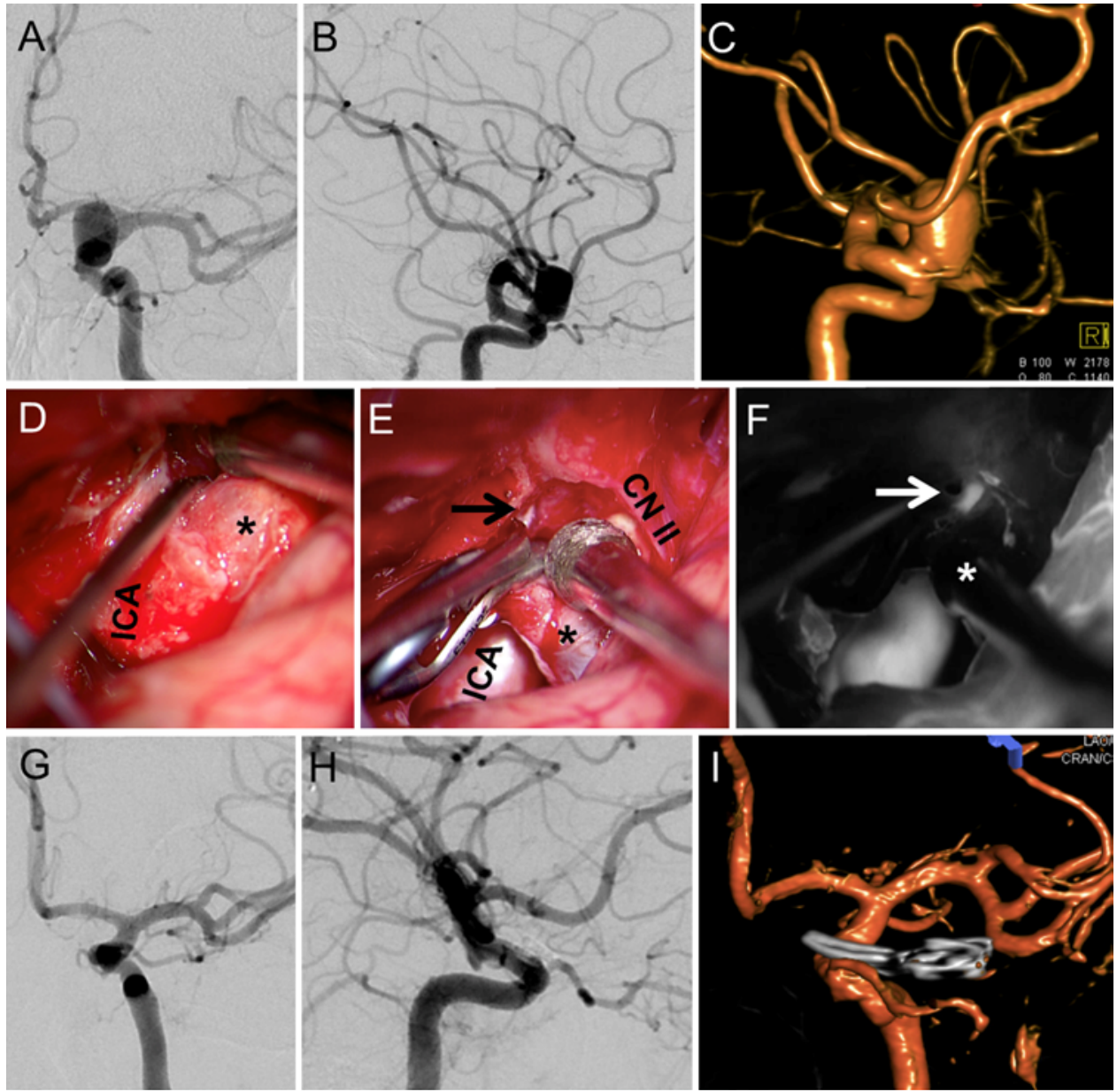

FIG. 2. Illustrative case 2. This 53-year-old woman presented with vision loss in her left eye and was diagnosed with a $6.8 \times 9.5-\mathrm{mm}$ unruptured left OphA aneurysm $(\mathbf{A}-\mathbf{C})$. Due to the presenting symptoms with compression of the left optic nerve, microsurgical clipping and decompression of the optic nerve was indicated. After an extradural anterior clinoidectomy the dura was opened and the sylvian fissure widely split using microsurgical technique. The dura over the clinoid region was opened to allow communication between the intradural and extradural spaces. The distal dural ring was then carefully dissected and incised circumferentially to expose the clinoidal segment. The aneurysm neck was then in view (D). Dissection of the aneurysm away from the optic nerve revealed the medially displaced OphA. The neck of the aneurysm was then brought into view and the aneurysm (asterisk) was clipped with a slightly curved Yaşargil clip (E), preserving the OphA (arrow). ICG video angiography confirmed patency of the parent and branch vessels and no filling of the aneurysm (F). The patient recovered well from surgery, and postoperative angiography confirmed complete occlusion of the aneurysm $(\mathbf{G}-\mathbf{I})$. However, this patient developed delayed worsening of her preexisting visual deficit.

SD or the median value plus range, as appropriate. Univariate logistic regression and the chi-square test or Fisher's exact test were performed to evaluate covariates for binary categorical dependent variables as appropriate. Normality of the data were evaluated with the Shapiro-Wilk test. Variables with non-normal distribution were compared by using the Mann-Whitney U-test. Multivariable logistic regression analysis was performed to evaluate significant correlation of postoperative visual deficit with procedurerelated factors. Only variables with values of $p<0.20$ in the univariate analysis were included in the multivariable logistic regression model-building process. Models were built by using forward/backward stepwise logistic regression with variables entered into the model and removed at the 0.20 significance level. Values of $p<0.05$ were considered statistically significant. Odds ratios are presented with $95 \%$ confidence intervals. The commercially avail- able software IBM SPSS (version 23; IBM Corp.) was used for all statistical analysis.

\section{Results}

A total of 220 patients with $230 \mathrm{OphA}$ aneurysms were treated microsurgically by the senior author (M.T.L.) between December 1997 and July 2016. These OphA aneurysms accounted for $6 \%$ of all aneurysms treated microsurgically during the same period (230/3942). The present study excluded 22 aneurysms - those treated with trapping with high-flow bypass $(n=4)$ or wrapping $(n=5)$, those that were unclippable $(\mathrm{n}=3)$, and those from cases with incomplete data $(\mathrm{n}=10)$. Therefore, 198 patients with 208 OphA aneurysms were included in the study. Overall, 41 aneurysms were large or giant in size $(20 \%)$ (Table 1). Twenty-three aneurysms (11\%) were ruptured, 156 (75\%) 


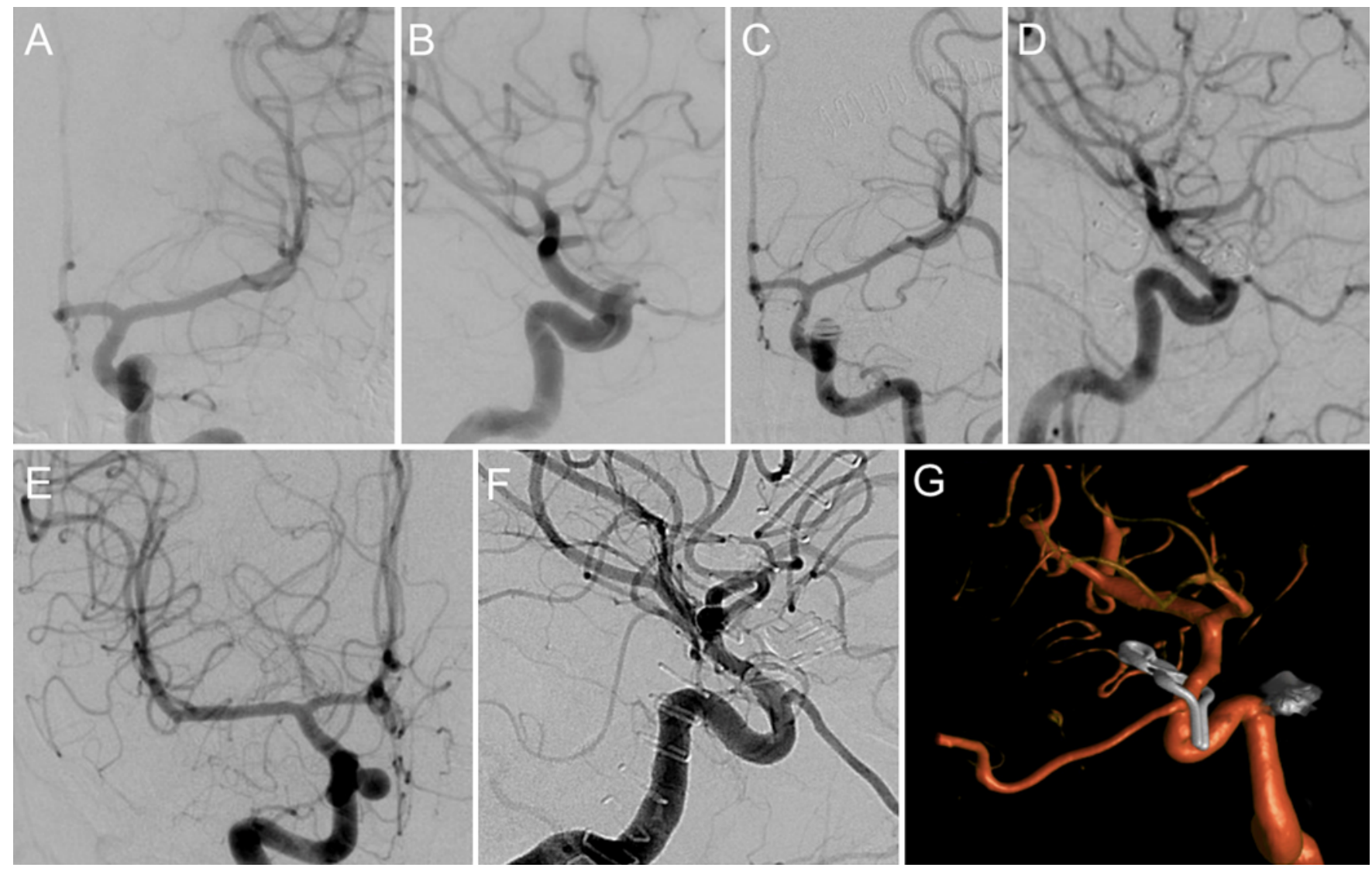

FIG. 3. Illustrative case 3. This 39-year-old woman with a family history of aneurysmal SAH sought medical attention when she developed headaches. MRI was suspicious for intracranial aneurysm. Catheter angiography confirmed an unruptured left OphA and an unruptured right carotid cave aneurysm (A, B, and E). The left OphA aneurysm was clipped through a left pterional mini-craniotomy with intradural anterior clinoidectomy. The distal dural ring was circumferentially incised to expose the aneurysm neck after anterior clinoidectomy. Then the neck was clipped with a slightly curved Yaşargil clip, which, because of the aneurysm's broad base, slid upward on the aneurysm to the top of the dome. An understacked curved clip was applied to grab more of the aneurysm tissue, and then an additional clip was applied under the second clip. This nicely reconstructed the neck and grabbed all of the aneurysmal tissue, while preserving the OphA. ICG video angiography and postoperative angiography confirmed patency of the carotid artery and no filling of the aneurysm ( $C$ and $\mathbf{D}$ ). After recovery from the surgery the patient underwent a right pterional craniotomy to treat the right carotid cave aneurysm. After extradural anterior clinoidectomy the proximal and distal ICA dural rings were opened and the aneurysm neck was dissected on both sides. With a $45^{\circ}$ angled fenestrated clip over the ICA and preserving the OphA, this aneurysm was nicely obliterated as confirmed with postoperative angiography ( $\mathbf{F}$ and $\mathbf{G})$.

were unruptured, and another 29 (14\%) were unruptured but associated with another ruptured aneurysm.

\section{Surgical Complications and Patient Outcomes}

Intraoperative rupture occurred in 6 microsurgical procedures (2.9\%). In 5 of these, the aneurysm was clipped successfully without postoperative neurological deficit. One patient's ruptured aneurysm (Hunt and Hess grade V) reruptured during dural opening and was clipped rapidly, but he developed severe brain swelling after the surgery and died.

Clinical outcome was as follows: $\mathrm{mRS}$ score 0 in 161 cases; $\mathrm{mRS}$ score 1 in 26 ; $\mathrm{mRS}$ score 2 in 13 ; $\mathrm{mRS}$ score 3 in 3; mRS score 4 in 3; mRS score 5 in 1 ; and $\mathrm{mRS}$ score 6 in 1 . Good outcomes (mRS score $0-2$ ) were observed in 200 cases $(96.2 \%)$ and poor (mRS score $3-6)$ in $8(3.8 \%)$. In the 8 cases with poor outcomes, 7 patients presented with significant deficits resulting from aneurysm rupture and were unchanged ( 3 patients with Hunt and Hess grade III subarachnoid hemorrhage [SAH] did not improve to independent living [mRS score 3], and the other 4 patients had poor Hunt and Hess grades at presentation [grade IV in 1 case and grade $\mathrm{V}$ in 3]). In 1 case, the patient presented with Hunt and Hess grade II SAH but the patient's condition deteriorated to mRS score 4 due to severe vasospasm.

Analysis of data from the 156 cases in which the patients presented without SAH and with unruptured aneurysms showed postoperative improvement in 12 cases, no change in clinical condition in 120 cases, and worsened clinical condition in 24 cases. All observed deteriorations were within the range of mRS scores for good outcomes (Table 2). Visual deficit was the cause of neurological worsening in 22 cases and postoperative infarction in 2.

\section{Visual Outcomes}

Seventeen patients presented with preoperative vi- 
TABLE 1. Summary of patient and aneurysm characteristics in 198 patients with 208 OphA aneurysms

\begin{tabular}{lc}
\hline \multicolumn{1}{c}{ Characteristic } & Value \\
\hline Mean age \pm SD, yrs & $52 \pm 14$ \\
\hline Sex & $34 / 198(17.2)$ \\
\hline Male & $164 / 198(82.8)$ \\
\hline Female & $17 / 198(8.6)$ \\
\hline Preop visual deficit & \\
\hline Aneurysm characteristics & \\
\hline Rupture status & $23 / 208(11.1)$ \\
\hline Ruptured & $156 / 208(75.0)$ \\
\hline Unruptured & $29 / 208(13.9)$ \\
\hline Unruptured w/ SAH due to another aneurysm \\
\hline Size & $167 / 208(80.3)$ \\
\hline Regular $(<12 \mathrm{~mm})$ & $31 / 208(14.9)$ \\
\hline Large (12-25 mm) & $10 / 208(4.8)$ \\
\hline Giant (>25 mm) & $18 / 208(8.7)$ \\
\hline Calcification & $13 / 208(6.3)$ \\
\hline Thrombosis & $67 / 208(32.2)$ \\
\hline Side & $141 / 208(67.8)$ \\
\hline Right
\end{tabular}

Data are $n(\%)$ unless otherwise indicated.

sual deficits, which included decreased visual acuity in $12(70.6 \%)$, visual field deficit in 4 (23.5\%), and both in 1 (5.9\%). Postoperatively, visual deficits improved in 9 $(52.9 \%)$ of these cases, remained stable in 5, and worsened in 3 (Table 3).

New postoperative visual deficit (including worsening of a preoperative visual deficit) at discharge was observed in $26(12.5 \%)$ of 208 cases. Patients with new visual deficits were managed with hypertensive therapy (systolic blood pressure greater than $160 \mathrm{~mm} \mathrm{Hg}$ ) and intravenous (IV) steroid treatment (dexamethasone [Decadron], $10 \mathrm{mg}$ IV every 6 hours), and the deficits disappeared completely in 4 cases. Visual deficits were found to have persisted in 22 cases $(10.6 \%)$ at the last follow-up (mean duration 11.1 months, range 1-96 months), with deficits consisting of decreased visual acuity in 5 patients (2.4\%), visual field defect in 8 patients (3.8\%; hemianopsia in 4 and inferior quadrantanopsia in 4), and monocular blindness in 9 (4.3\%). According to univariate logistic regression analysis, risk factors for new postoperative visual deficit included anterior clinoidectomy (OR 10.9, 95\% CI 1.45-82.6, $\mathrm{p}=0.02)$, use of multiple clips (OR 3.04.95\% CI 1.18-7.84, $\mathrm{p}=0.02$ ), and aneurysm calcification (OR 3.10, 95\% CI 1.00-9.56, p = 0.04) (Table 4). A multivariable logistic regression model was built including fenestrated clip use and a contralateral approach, but it revealed marginally significant correlation. In the final multivariable analysis, only anterior clinoidectomy (OR 8.76, 95\% CI 1.14-67.1, $\mathrm{p}=0.04)$ remained a significant predictor for postoperative visual deficit (Table 5).

Seven patients developed delayed postoperative visual deficit more than 1.5 days after surgery (Table 6). Five $(71 \%)$ of these patients awoke from surgery with intact vision and developed delayed vision loss. An immediate postoperative visual deficit worsened in a delayed manner in 1 patient (illustrative case 2). None of the patients with delayed visual deficits demonstrated any improvement in those deficits during a mean follow-up of 7.7 months (range 2-18 months). Comparison of immediate and delayed postoperative visual deficits identified the use of a fenestrated clip as a significant factor for delayed deficits (Table 7).

\section{Aneurysm Outcomes}

Catheter angiography was performed postoperatively in 191 (91.8\%) of 208 cases. Among these cases, strict aneurysm exclusion was confirmed in 174 (91.1\%). Small neck remnants were observed in 17 cases $(8.9 \%)$, none of them significant and none requiring additional treatment. $\mathrm{Pa}-$ tency of the OphA was observed in all but 1 case (99.5\%), and in that case the lack of patency did not cause a visual deficit.

All patients were advised to return for follow-up angiography 5 years after treatment. Overall, 32 (16\%) of 198 patients underwent catheter angiography for long-term follow-up at a mean of 6.4 years after aneurysm treatment. One small aneurysm recurrence was observed (3.1\%), less than $2 \mathrm{~mm}$ in size and associated with a giant aneurysm. This recurrence did not require treatment and is under ob-

TABLE 2. Surgical results, complications, and functional outcome

\begin{tabular}{|c|c|c|c|c|}
\hline Variable & Total $(n=208)$ & Ruptured $(n=23)$ & Unruptured $(n=156)$ & Unruptured $(\mathrm{SAH})^{*}(\mathrm{n}=29)$ \\
\hline \multicolumn{5}{|l|}{ Aneurysm obliteration $†$} \\
\hline Complete & $174(91.1)$ & $16(80.0)$ & $134(92.4)$ & $24(92.3)$ \\
\hline Neck remnant & $17(8.9)$ & $4(20.0)$ & $11(7.6)$ & $2(7.7)$ \\
\hline Intraop rupture & $6(2.9)$ & $2(8.7)$ & $0(0)$ & $4(13.8)$ \\
\hline Postop infarction & $13(6.3)$ & $3(13.0)$ & $6(3.8)$ & $4(13.8)$ \\
\hline \multicolumn{5}{|c|}{ Functional outcome (at 6 wks) } \\
\hline Good (mRS score $0-2$ ) & $200(96.2)$ & $19(82.6)$ & $156(100)$ & $25(86.2)$ \\
\hline Poor (mRS score 3-6) & $8(3.8)$ & $4(17.4)$ & $0(0)$ & $4(13.8)$ \\
\hline
\end{tabular}


TABLE 3. Characteristics and visual outcome in patients with preoperative visual symptoms

\begin{tabular}{lcccc}
\hline \multicolumn{1}{c}{ Variable } & Total $(\mathrm{n}=17)$ & Improved $(\mathrm{n}=9)$ & Unchanged or Worsened $(\mathrm{n}=8)$ & $\mathrm{p}$ Value \\
\hline Mean age \pm SD, yrs & $50.5 \pm 13.2$ & $49.8 \pm 14.5$ & $51.3 \pm 12.4$ & 0.84 \\
\hline Female sex & $15(88.2)$ & $8(88.9)$ & $7(87.5)$ & 0.74 \\
\hline Visual symptom & & & \\
\hline \multicolumn{1}{c}{ Decreased visual acuity } & $12(70.6)$ & $7(77.8)$ & $5(62.5)$ & 0.44 \\
\hline$\quad$ Visual field deficit & $4(23.5)$ & $1(11.1)$ & $3(37.5)$ & 0.24 \\
\hline$\quad$ Decreased visual acuity + visual field deficit & $1(5.9)$ & $1(11.1)$ & $0(0)$ & 0.53 \\
\hline Aneurysm characteristic & & & $13.2(2-42)$ & 0.65 \\
\hline$\quad$ Median size, mm (range) & $14.6(2-42)$ & $15.8(3-30)$ & $0(0)$ & 0.53 \\
\hline Calcification & $1(5.9)$ & $1(11.1)$ & $1(12.5)$ & 0.55 \\
\hline$\quad$ Thrombosis & $3(17.6)$ & $2(22.2)$ & $1(12.5)$ & 0.34 \\
\hline Onset-op interval w/in 3 mos & $4(23.5)$ & $3(33.3)$ & & \\
\hline
\end{tabular}

Data are $\mathrm{n}(\%)$ unless otherwise indicated.

servation. Included in these 32 patients were 3 other patients with small, intentional residual aneurysms that remained stable in size and were observed.

\section{Discussion}

\section{Microsurgical Clipping of OphA Aneurysms}

This experience with 208 OphA aneurysms in 198 patients demonstrates the efficacy and durability of surgical clipping in the management of these lesions. Despite 20\% of these aneurysms being large or giant in size, $9 \%$ calcified, and $6 \%$ thrombotic, angiographically complete aneurysm occlusion was accomplished in $91 \%$ of aneurysms, with OphA patency preserved in $99.5 \%$. The aneurysm recurrence rate was $3.1 \%$, and the retreatment rate was $0 \%$. Good outcomes were observed in $96.2 \%$ of cases, and poor outcomes were due entirely to the initial aneurysm rupture, not the surgical procedure. In $85 \%$ of cases in which pa- tients presented with an unruptured OphA aneurysm, the patients' clinical condition improved or remained stable. The cause of worsening in most remaining cases, and the drawback of clipping OphA aneurysms generally, was new visual deficits. While surgical clipping enabled aneurysm deflation and optic nerve decompression that resulted in visual improvements in over half of our patients with preoperative visual deficits, it caused a new visual field defect (hemianopsia or quadrantanopsia) in 3.8\% and monocular blindness in $4.3 \%$.

Visual morbidity after microsurgical clipping is well reported in the literature and ranges from $0 \%$ to $28.5 \%$ (Table 8). $4,12,15,16,18,19,22-25,27,34$ According to logistic regression analysis, anterior clinoidectomy was the only factor associated with new postoperative deficit, but anterior clinoidectomy is a necessary part of clipping OphA aneurysms. Incomplete anterior clinoidectomy is not a viable solution,

TABLE 4. Univariate analysis of factors related to new visual deficit

\begin{tabular}{|c|c|c|c|c|c|}
\hline \multirow[b]{2}{*}{ Variable } & \multicolumn{2}{|c|}{ Postop Visual Deficit } & \multicolumn{3}{|c|}{ Univariate } \\
\hline & Absent $(n=182)$ & Present $(n=26)$ & OR & $95 \% \mathrm{Cl}$ & p Value \\
\hline \multicolumn{6}{|l|}{ Aneurysm characteristic } \\
\hline Ruptured & $21(11.5)$ & $2(7.7)$ & 1.43 & $0.35-5.74$ & 0.62 \\
\hline Size $>12 \mathrm{~mm}$ & $35(19.2)$ & $4(15.4)$ & 0.79 & $0.30-2.12$ & 0.65 \\
\hline Calcification & $13(7.1)$ & $5(19.2)$ & 3.10 & $1.00-9.55$ & 0.04 \\
\hline Thrombosis & $12(6.6)$ & $1(3.8)$ & 2.43 & $0.31-19.1$ & 0.40 \\
\hline Anterior clinoidectomy & $132(72.5)$ & $25(96.2)$ & 10.9 & $1.45-82.6$ & 0.02 \\
\hline Multiple clips & $106(58.2)$ & $21(80.8)$ & 3.04 & $1.18-7.84$ & 0.02 \\
\hline \multicolumn{6}{|l|}{ Clip type } \\
\hline Straight & $111(61.0)$ & $16(61.5)$ & 1.16 & $0.51-2.60$ & 0.73 \\
\hline Fenestrated & $55(30.2)$ & $13(50.0)$ & 2.19 & $0.98-4.90$ & 0.06 \\
\hline Curved & $60(33.0)$ & $11(42.3)$ & 1.68 & $0.75-3.76$ & 0.21 \\
\hline Angled & $33(18.1)$ & $4(15.4)$ & 1.06 & $0.38-2.99$ & 0.91 \\
\hline Complete exclusion* & $151(91.5)$ & $23(88.5)$ & 1.41 & $0.38-5.28$ & 0.61 \\
\hline Contralateral clipping & $37(20.3)$ & $2(7.7)$ & 0.31 & $0.07-1.34$ & 0.12 \\
\hline
\end{tabular}

Data are $\mathrm{n}(\%)$. Boldface type indicates statistical significance.

* Aneurysm obliteration was evaluated in 191 cases in which postoperative catheter angiography was performed. 
TABLE 5. Multivariate logistic regression analysis of factors related to new visual deficit

\begin{tabular}{lccc}
\hline \multicolumn{1}{c}{ Variable } & OR & $95 \% \mathrm{Cl}$ & $\mathrm{p}$ Value \\
\hline Anterior clinoidectomy & 8.76 & $1.14-67.1$ & $\mathbf{0 . 0 4}$ \\
\hline Aneurysm calcification & 2.89 & $0.91-9.18$ & 0.07 \\
\hline Multiple clips & 2.08 & $0.73-5.99$ & 0.17 \\
\hline
\end{tabular}

Boldface type indicates statistical significance.

because complete removal of this structure is needed to dissect the clinoidal segment of the ICA, incise the distal dural ring, and expose the proximal aneurysm neck adequately. Possible mechanisms of visual morbidity include thermal or vibrational injury of the optic nerve from the high-speed drill during drilling of the anterior clinoid process and its attachments, optic nerve manipulation during dissection from the aneurysm, optic pathway ischemia from perforator occlusion or spasm, and direct optic nerve compression by the applied clip..$^{3,11}$ Deliberate precautions can be taken to minimize these risks to the optic nerve.

Use of the high-speed drill cannot be eliminated from anterior clinoidectomy. Other devices, such as ultrasonic aspirators, were tried during this experience in an effort to reduce thermal and vibrational injury, but the precision of these devices was inferior to that of a drill and they failed to reduce visual morbidity. The optic roof, medial sphenoid ridge, and optic strut must be drilled with generous cold irrigation and frequent pauses to dissipate heat. The touch with the drill should be light. The optic canal should be unroofed early and widely, with bone around the optic canal thinned and fractured away from the nerve to avoid drilling next to the nerve.

A thorough knowledge of the anatomy of the anterior clinoid process, distal dural ring, paraclinoid ICA, and cavernous sinus is the best defense against optic nerve injury because it informs the dissection. This knowledge is gained through practice with cadaveric specimens that teach real anatomy without the challenges of bleeding or threat of morbidity. Fibrin glue was used in the latter half of our surgical experience to control bleeding from the cavernous sinus during anterior clinoidectomy and dural ring dissection. Previously, applying oxidized cellulose
TABLE 7. Patients with immediate and delayed postoperative visual deficit

\begin{tabular}{|c|c|c|c|}
\hline \multirow[b]{2}{*}{ Characteristic } & \multicolumn{2}{|c|}{ Visual Deficit* } & \multirow[b]{2}{*}{$\begin{array}{c}p \\
\text { Value }\end{array}$} \\
\hline & $\begin{array}{l}\text { Immediate } \\
(n=20)\end{array}$ & $\begin{array}{c}\text { Delayed } \\
(n=7)\end{array}$ & \\
\hline Mean age $\pm S D$, yrs & $55.3 \pm 12.7$ & $50.1 \pm 8.3$ & 0.52 \\
\hline Female sex & $17(85)$ & $7(100)$ & 0.31 \\
\hline Visual deficit & & & 0.48 \\
\hline Visual acuity & $12(60)$ & $5(71.4)$ & \\
\hline Visual field & $8(40)$ & $2(28.6)$ & \\
\hline \multicolumn{4}{|l|}{ Aneurysm characteristic } \\
\hline Rupture & $2(10)$ & $0(0)$ & 0.47 \\
\hline Left sided & $16(80)$ & $5(71.4)$ & 0.50 \\
\hline Size $>12 \mathrm{~mm}$ & $3(15)$ & $1(14.3)$ & 0.73 \\
\hline Calcification & $4(20)$ & $1(14.3)$ & 0.61 \\
\hline Thrombosis & $1(5)$ & $0(0)$ & 0.74 \\
\hline Anterior clinoidectomy & $19(95)$ & $7(100)$ & 0.47 \\
\hline Multiple clips & $15(75)$ & $6(85.7)$ & 0.50 \\
\hline \multicolumn{4}{|l|}{ Clip type } \\
\hline Straight & $13(65)$ & $3(42.9)$ & 0.28 \\
\hline Fenestrated & $7(35)$ & $6(85.7)$ & 0.03 \\
\hline Curved & $9(45)$ & $2(28.6)$ & 0.38 \\
\hline Angled & $2(10)$ & $2(28.6)$ & 0.27 \\
\hline Visual improvement (at last follow-up) & $4(20)$ & $0(0)$ & 0.28 \\
\hline
\end{tabular}

Data are $n(\%)$ unless otherwise indicated. Boldface type indicates statistical significance.

* One patient developed immediate and delayed postoperative visual deficit and is included in both groups.

and pressure controlled the bleeding, but incompletely, which limited further dissection. The injection of fibrin glue into the cavernous sinus through a perforation in the carotid-oculomotor membrane provided complete control of bleeding, which facilitated thorough dissection of the aneurysm. Improved hemostasis and aggressive aneurysm dissection made possible by casting the cavernous sinus with fibrin glue facilitated microsurgical clipping, potentially contributing to improved results.

TABLE 6. Summary of cases with postoperative delayed visual deficit*

\begin{tabular}{|c|c|c|c|c|c|c|c|c|c|c|c|}
\hline \multirow[b]{2}{*}{ Case } & \multirow{2}{*}{$\begin{array}{l}\text { Age (yrs), } \\
\text { Sex }\end{array}$} & \multirow{2}{*}{$\begin{array}{l}\text { Visual } \\
\text { Deficit }\end{array}$} & \multicolumn{3}{|c|}{ Aneurysm Characteristics } & \multirow{2}{*}{$\begin{array}{c}\text { Op-Onset } \\
\text { Interval (days) }\end{array}$} & \multicolumn{4}{|c|}{ Clip Type } & \multirow{2}{*}{$\begin{array}{c}\text { Visual } \\
\text { Outcome } †\end{array}$} \\
\hline & & & Size $(\mathrm{mm})$ & Calcification & Thrombosis & & Straight & Fenestrated & Curved & Angled & \\
\hline 1 & $43, \mathrm{~F}$ & Vision loss & 13 & - & - & Unknown & 1 & 1 & 0 & 0 & Unchanged \\
\hline 2 & $50, \mathrm{~F}$ & Visual field & 5 & - & - & 3 & 0 & 0 & 1 & 0 & Unchanged \\
\hline 3 & $52, F$ & Vision loss & 8 & - & - & 2 & 0 & 1 & 0 & 1 & Unchanged \\
\hline 4 & $50, F$ & Vision loss & 10 & - & - & 1.5 & 1 & 1 & 1 & 0 & Unchanged \\
\hline 5 & $67, \mathrm{~F}$ & Vision loss & 7 & + & - & Unknown & 1 & 1 & 0 & 0 & Unchanged \\
\hline 6 & $47, \mathrm{~F}$ & Visual field & 6 & - & - & 1.5 & 0 & 1 & 0 & 1 & Unchanged \\
\hline 7 & $42, F$ & Vision loss & 4 & - & - & 2 & 0 & 1 & 1 & 0 & Unchanged \\
\hline
\end{tabular}

- = absent; + = present.

Data are numbers of cases unless otherwise indicated.

* One patient (case 2) developed immediate and delayed postoperative visual deficit.

$\dagger$ At last follow-up. 
TABLE 8. Visual outcome after microsurgical treatment of paraclinoid aneurysms

\begin{tabular}{|c|c|c|c|c|c|c|}
\hline \multirow[b]{2}{*}{ Authors \& Year } & \multirow{2}{*}{$\begin{array}{l}\text { No. of Treated } \\
\text { Aneurysms }\end{array}$} & \multicolumn{4}{|c|}{ Cases w/ Preop Visual Deficit } & \multirow{2}{*}{$\begin{array}{l}\text { New Postop } \\
\text { Visual Deficit }\end{array}$} \\
\hline & & Total & Improved & Unchanged & Worsened & \\
\hline Day, 1990 & 54 & 23 & $17(73.9)$ & $6(26.1)$ & $0(0)$ & $3(5.6)$ \\
\hline Hoh et al., 2001 & 180 & 12 & $8(66.7)$ & $3(25.0)$ & $1(8.3)$ & $5(2.8)$ \\
\hline Raco et al., 2008 & 108 & 26 & $13(50.0)$ & $12(46.2)$ & $1(3.8)$ & $3(2.8)$ \\
\hline Fulkerson et al., 2009* & 126 & 27 & NR & NR & NR & $6(4.8)$ \\
\hline Nanda \& Javalkar, 2011 & 86 & 15 & $10(66.7)$ & $3(20.0)$ & $2(33.3)$ & $7(8.1)$ \\
\hline Kanagalingam et al., 2012 & 69 & 6 & $0(0)$ & $1(16.7)$ & $5(83.3)$ & $30(29.7) \dagger$ \\
\hline Lai \& Morgan, 2013 & 182 & 28 & $16(57.1)$ & $9(32.1)$ & $3(10.7)$ & $6(3.3) \ddagger$ \\
\hline Matsukawa et al., 2016 & 136 & 2 & $2(100)$ & $0(0)$ & $0(0)$ & $23(16.9)$ \\
\hline Kikuta et al., 2016 & 38 & 18 & NR & NR & NR & $10(26.3)$ \\
\hline Pasqualin et al., 2016 & 66 & 20 & $3(15.0)$ & $10(50.0)$ & $7(35.0)$ & $13(19.7)$ \\
\hline Present series, $2017^{*}$ & 208 & 17 & $9(52.9)$ & $5(29.4)$ & $3(17.6)$ & $22(10.6)$ \\
\hline
\end{tabular}

The arachnoid membrane forming the lateral wall of the carotid cistern and enveloping the optic nerve can be preserved for the drilling, which keeps bone dust, blood, fibrin glue, and other debris from contacting the nerve. Even with a full sylvian fissure split, this protective arachnoid layer can be preserved. Even after clinoidectomy is completed, this layer is maintained as long as possible to protect the nerve and its delicate perforators. In addition, the dissection should depress the carotid artery rather than lift the optic nerve. A "no-touch" policy toward the optic nerve helps keep instruments and pressure off the nerve. Perforators to the optic nerve from the superior hypophyseal artery and directly from the ICA are fragile and must be handled delicately. As our experience demonstrates, the OphA can be preserved reliably with clipping and is usually intact in patients with new visual deficits. Therefore, the perforators in the subarachnoid spaces around the aneurysm-not the OphA-are responsible for vascularmediated visual complications.

Aneurysm clips might also contribute to visual morbidity after clipping. The medial blade of the clip or clips must be applied meticulously to spare perforators coursing to the undersurface of the optic nerve. When we analyzed differences between immediate and delayed postoperative visual deficits, only the use of a fenestrated clip was significant $(p<0.03)$. The fenestrated clip was used as part of tandem clipping, which implies a broad-neck aneurysm or a calcified, atherosclerotic neck. The fenestrated clip also required the use of a second closing clip, which might create upward pressure on the underside of the optic nerve and impaired microcirculation. While the exact mechanism of delayed visual deficits is unclear, these observations suggest delayed vasospasm or vascular compromise from mechanical pressure, as from a clip, causing optic nerve ischemia.

We operate on OphA aneurysms blinded to the patient's possible blindness. Intraoperative visual evoked responses might provide feedback for the neurosurgeon during the dissection and clip application, but this technique has not been reliable. New monocular blindness is one of the neurosurgeon's worst complications and the patient's most distressing deficits. Patients react strongly to this problem, even though they can see with the other eye. Therefore, every precaution possible must be taken when drilling around optic canal, manipulating the optic nerve during aneurysm dissection, or handling its perforators.

\section{Endovascular Therapy for OphA Aneurysms}

Although favorable outcomes have been reported for surgical treatment of OphA aneurysms in this and previous studies, ${ }^{2,10,20,21,23,27}$ advances in endovascular techniques and technology have increased the popularity of endovascular therapy for paraclinoid aneurysms. ${ }^{8,10,11,29,31}$ However, endovascular treatment is associated with a lower rate of complete aneurysm occlusion and more frequent recurrences that might lead to hemorrhage or necessitate retreatment. ${ }^{6,31}$ Some authors have reported excellent results using flow diverters, ${ }^{1}$ but their use is controversial because of the associated morbidity and even mortality. Visual outcome after endovascular treatment is also a concern. Previous studies reported a $6 \%$ rate of new visual disturbance or worsening of existing visual symptoms after endovascular therapy, $, 12,14,15,30$ leading to the conclusion that visual outcomes are equivalent after microsurgical clipping and endovascular coiling. ${ }^{12,32}$ Recent reports of visual morbidity associated with flow diverters are mixed., ${ }^{1,26,28}$ While Burrows et al. ${ }^{1}$ reported no permanent visual deficits in their experience, Rouchaud et al. ${ }^{28}$ found that $39.3 \%$ experienced visual complications, and patients with the OphA originating from the aneurysm sac were at high risk of retinal emboli ( $80 \%$ ). Further studies are needed to determine the visual morbidity of endovascular therapy for OphA aneurysms, particularly with flow diverters.

The prevalence of pretreatment visual symptoms has been reported to be $7 \%-32 \%$ in patients with unruptured paraclinoid aneurysms. , $^{2,10,12,15,20,23,27}$ Previous studies have demonstrated improvement or resolution of visual symp- 
toms in $0 \%-75 \%$ of patients treated microsurgically., 3,12,15,20, $23,24,27,30$ The literature provides evidence that microsurgery is superior to endovascular therapy with regard to resolution of preoperative visual deficits, due to the ability to puncture or resect the aneurysm after clipping and thereby relieve its mass effect on the anterior visual pathways. . $^{3,23,33}$ Date et al. ${ }^{3}$ reported that preoperative visual symptoms improved when the target aneurysm was treated within 3 months of visual symptom onset. In this series, patients had visual deficits at initial presentation in 17 (8.2\%) of 208 cases, and in $9(52.9 \%)$ of these cases, the patients experienced improvement or resolution, which is within the range of previous studies (Table 8). Four (23.5\%) of these patients were treated within 3 months, and $3(75 \%)$ of these patients had improved visual outcome postoperatively, consistent with the observation that patients with preexisting visual deficits optimize their outcome when treated expeditiously.

In our series, delayed visual deficit was noted after 7 microsurgical procedures (3.4\%). While most visual complications after microsurgical clipping of paraclinoid aneurysms are due to an intraoperative action and are apparent immediately after the procedure, delayed visual deficits suggest something different, more likely a vascular event rather than a thermal, vibrational, or mechanical injury. Recently, Matsukawa et al. ${ }^{22}$ reported that $7.4 \%$ of patients with unruptured paraclinoid aneurysms developed delayed visual deficits 12-72 hours after surgery. The authors suggested the disturbance of the superior ophthalmic vein or vasospasm of the superior hypophyseal artery was the cause. In our study, all but one of the patients with a delayed visual deficit had small or medium-sized aneurysms, and no patient showed improvement with medical treatment. The exact mechanism is unclear, but we agree that delayed visual deficits are likely microvascular in origin, due to perforator mismanagement or delayed vasospasm that results in optic nerve ischemia. Despite the lack of conclusive evidence, these observations encouraged us to try treating all patients prophylactically with hypertension, hypervolemia, and high-dose corticosteroid therapy during the first 48 hours after anterior clinoidectomy, rather than treating only those who become symptomatic later. Anecdotally, this proactive rather than reactive approach appears to be helping to reduce the incidence of visual complications in patients with clipped OphA aneurysms, but more data collection and a thorough analysis will be needed to determine efficacy.

\section{Limitations}

The present study has several limitations. First, it is a retrospective analysis, despite being based on a prospectively maintained database, and there was no control group with observed or endovascular treated OphA aneurysms. Complete data were not available for 10 aneurysms treated by microsurgical clipping, and these patients were excluded. Patients included in this series reflected selection and referral biases inherent in a tertiary care practice at an academic medical center. Second, this might be the largest, consecutive, single-surgeon experience reported for OphA aneurysms, but operative techniques, technologies, and competencies undoubtedly changed over this 19-year period, and this may have impacted outcomes.
Third, formal neuro-ophthalmological evaluation was performed in most, but not all cases. Determination of new visual deficits postoperatively in patients not seen by an ophthalmologist was qualitative, especially changes in visual acuity. In each of these cases, visual assessment was performed by a neurologist and a neurosurgeon and was performed repeatedly during hospitalization and at every follow-up visit. We deliberately included any and all visual deficits, including changes in visual acuity, in an attempt to increase the study's sensitivity to visual complications. This approach may overestimate our visual complication rate relative to other reports with less stringent criteria. Still, the $3.8 \%$ risk of a new visual field defect (hemianopsia or quadrantanopsia) and the $4.3 \%$ risk of monocular blindness are useful figures to discuss with patients as they weigh the relative risks of microsurgical, endovascular, and conservative management. Finally, although this study demonstrates the efficacy of microsurgical clipping for OphA aneurysms with good clinical outcomes, further studies, in particular, randomized control trials, are needed to establish the superiority of microsurgical clipping over endovascular management or vice versa.

\section{Conclusions}

This experience with 208 OphA aneurysms in 198 patients demonstrates the efficacy and durability of microsurgical clipping. Complete aneurysm occlusion was accomplished in $91 \%$ of aneurysms, with OphA patency preserved in $99.5 \%$ of cases, aneurysm recurrence occurring in $3.1 \%$, and retreatment required in $0 \%$. Good outcomes were observed in $96.2 \%$ of patients, and poor outcomes were due entirely to the initial aneurysm rupture. The most important risk associated with clipping OphA aneurysms was a new visual deficit, specifically a visual field defect (hemianopsia or quadrantanopsia) in $3.8 \%$, decreased visual acuity in $2.4 \%$, and monocular blindness in 4.3\%. Microsurgical clipping enabled aneurysm deflation and optic nerve decompression that resulted in visual improvements in over half of patients with preoperative visual deficits, demonstrating the success of this treatment modality in this subgroup. Meticulous microsurgical technique is necessary during anterior clinoidectomy, aneurysm dissection, and clip application to optimize visual outcomes, and aggressive medical management postoperatively might decrease the incidence of delayed visual deficits. As the results of endovascular therapy and specifically flow diverter treatment become known, they warrant comparison with these surgical benchmarks to determine best practices.

\section{References}

1. Burrows AM, Brinjikji W, Puffer RC, Cloft H, Kallmes DF, Lanzino G: Flow diversion for ophthalmic artery aneurysms. AJNR Am J Neuroradiol 37:1866-1869, 2016

2. Colli BO, Carlotti CG Jr, Assirati JA Jr, Abud DG, Amato MC, Dezena RA: Results of microsurgical treatment of paraclinoid carotid aneurysms. Neurosurg Rev 36:99-115, 2013

3. Date I, Asari S, Ohmoto T: Cerebral aneurysms causing visual symptoms: their features and surgical outcome. Clin Neurol Neurosurg 100:259-267, 1998

4. Day AL: Aneurysms of the ophthalmic segment. A clinical and anatomical analysis. J Neurosurg 72:677-691, 1990 
5. Drake CG, Vanderlinden RG, Amacher AL: Carotid-ophthalmic aneurysms. J Neurosurg 29:24-31, 1968

6. D’Urso PI, Karadeli HH, Kallmes DF, Cloft HJ, Lanzino G: Coiling for paraclinoid aneurysms: time to make way for flow diverters? AJNR Am J Neuroradiol 33:1470-1474, 2012

7. D'Urso PI, Lanzino G, Cloft HJ, Kallmes DF: Flow diversion for intracranial aneurysms: a review. Stroke 42:2363-2368, 2011

8. Durst CR, Starke RM, Gaughen J, Nguyen Q, Patrie J, Jensen ME, et al: Vision outcomes and major complications after endovascular coil embolization of ophthalmic segment aneurysms. AJNR Am J Neuroradiol 35:2140-2145, 2014

9. Ferguson GG, Drake CG: Carotid-ophthalmic aneurysms: visual abnormalities in 32 patients and the results of treatment. Surg Neurol 16:1-8, 1981

10. Fulkerson DH, Horner TG, Payner TD, Leipzig TJ, Scott JA, DeNardo AJ, et al: Results, outcomes, and follow-up of remnants in the treatment of ophthalmic aneurysms: a 16-year experience of a combined neurosurgical and endovascular team. Neurosurgery 64:218-230, 2009

11. Heran NS, Song JK, Kupersmith MJ, Niimi Y, Namba K, Langer DJ, et al: Large ophthalmic segment aneurysms with anterior optic pathway compression: assessment of anatomical and visual outcomes after endosaccular coil therapy. J Neurosurg 106:968-975, 2007

12. Hoh BL, Carter BS, Budzik RF, Putman CM, Ogilvy CS: Results after surgical and endovascular treatment of paraclinoid aneurysms by a combined neurovascular team. Neurosurgery 48:78-90, 2001

13. Horiuchi T, Yamamoto Y, Suzuki Y, Kobayashi M, Ichinose S, Hongo K: Clipping surgery for paraclinoid carotid aneurysm. Acta Neurochir Suppl 123:27-32, 2016

14. Iihara K, Murao K, Sakai N, Shindo A, Sakai H, Higashi $\mathrm{T}$, et al: Unruptured paraclinoid aneurysms: a management strategy. J Neurosurg 99:241-247, 2003

15. Kanagalingam S, Gailloud P, Tamargo RJ, Subramanian PS, Miller NR: Visual sequelae after consensus-based treatment of ophthalmic artery segment aneurysms: the Johns Hopkins experience. J Neuroophthalmol 32:27-32, 2012

16. Kattner KA, Bailes J, Fukushima T: Direct surgical management of large bulbous and giant aneurysms involving the paraclinoid segment of the internal carotid artery: report of 29 cases. Surg Neurol 49:471-480, 1998

17. Khan N, Yoshimura S, Roth P, Cesnulis E, Koenue-Leblebicioglu D, Curcic M, et al: Conventional microsurgical treatment of paraclinoid aneurysms: state of the art with the use of the selective extradural anterior clinoidectomy SEAC. Acta Neurochir Suppl 94:23-29, 2005

18. Kikuta K, Kitai R, Kodera T, Arishima H, Isozaki M, Hashimoto N, et al: Predictive factors for the occurrence of visual and ischemic complications after open surgery for paraclinoid aneurysms of the internal carotid artery. Acta Neurochir Suppl 123:41-49, 2016

19. Kumon Y, Sakaki S, Kohno K, Ohta S, Ohue S, Oka Y: Asymptomatic, unruptured carotid-ophthalmic artery aneurysms: angiographical differentiation of each type, operative results, and indications. Surg Neurol 48:465-472, 1997

20. Lai LT, Morgan MK: Outcomes for unruptured ophthalmic segment aneurysm surgery. J Clin Neurosci 20:1127-1133, 2013

21. Matano F, Tanikawa R, Kamiyama H, Ota N, Tsuboi T, Noda $\mathrm{K}$, et al: Surgical treatment of 127 paraclinoid aneurysms with multifarious strategy: factors related with outcome. World Neurosurg 85:169-176, 2016

22. Matsukawa H, Tanikawa R, Kamiyama H, Tsuboi T, Noda K, Ota N, et al: Risk factors for visual impairments in patients with unruptured intradural paraclinoid aneurysms treated by neck clipping without bypass surgery. World Neurosurg 91:183-189, 2016
23. Nanda A, Javalkar V: Microneurosurgical management of ophthalmic segment of the internal carotid artery aneurysms: single-surgeon operative experience from Louisiana State University, Shreveport. Neurosurgery 68:355-371, 2011

24. Nonaka T, Haraguchi K, Baba T, Koyanagi I, Houkin K: Clinical manifestations and surgical results for paraclinoid cerebral aneurysms presenting with visual symptoms. Surg Neurol 67:612-619, 2007

25. Pasqualin A, Meneghelli P, Cozzi F, Chioffi F: Outcome after surgical treatment of paraclinoid carotid aneurysms. Acta Neurochir Suppl 123:33-39, 2016

26. Puffer RC, Kallmes DF, Cloft HJ, Lanzino G: Patency of the ophthalmic artery after flow diversion treatment of paraclinoid aneurysms. J Neurosurg 116:892-896, 2012

27. Raco A, Frati A, Santoro A, Vangelista T, Salvati M, Delfini $\mathrm{R}$, et al: Long-term surgical results with aneurysms involving the ophthalmic segment of the carotid artery. J Neurosurg 108:1200-1210, 2008

28. Rouchaud A, Leclerc O, Benayoun Y, Saleme S, Camilleri Y, D'Argento F, et al: Visual outcomes with flow-diverter stents covering the ophthalmic artery for treatment of internal carotid artery aneurysms. AJNR Am J Neuroradiol 36:330336,2015

29. Schmidt GW, Oster SF, Golnik KC, Tumialán LM, Biousse $\mathrm{V}$, Turbin R, et al: Isolated progressive visual loss after coiling of paraclinoid aneurysms. AJNR Am J Neuroradiol 28:1882-1889, 2007

30. Schuss P, Güresir E, Berkefeld J, Seifert V, Vatter H: Influence of surgical or endovascular treatment on visual symptoms caused by intracranial aneurysms: single-center series and systematic review. J Neurosurg 115:694-699, 2011

31. Shimizu K, Imamura H, Mineharu Y, Adachi H, Sakai C, Sakai N: Endovascular treatment of unruptured paraclinoid aneurysms: single-center experience with 400 cases and literature review. AJNR Am J Neuroradiol 37:679-685, 2016

32. Shimizu T, Naito I, Aihara M, Fujimaki H, Asakura K, Miyamoto N, et al: Visual outcomes of endovascular and microsurgical treatment for large or giant paraclinoid aneurysms. Acta Neurochir (Wien) 157:13-20, 2015

33. Tawk RG, Villalobos HJ, Levy EI, Hopkins LN: Surgical decompression and coil removal for the recovery of vision after coiling and proximal occlusion of a clinoidal segment aneurysm: technical case report. Neurosurgery 58:E1217, 2006

34. Yadla S, Campbell PG, Grobelny B, Jallo J, Gonzalez LF, Rosenwasser RH, et al: Open and endovascular treatment of unruptured carotid-ophthalmic aneurysms: clinical and radiographic outcomes. Neurosurgery 68:1434-1443, 2011

\section{Disclosures}

The authors report no conflict of interest concerning the materials or methods used in this study or the findings specified in this paper.

\section{Author Contributions}

Conception and design: Kamide. Acquisition of data: Kamide, Tabani, Safaee, Burkhardt. Analysis and interpretation of data: Kamide, Burkhardt. Drafting the article: Kamide, Tabani. Critically revising the article: Kamide, Burkhardt. Reviewed submitted version of manuscript: Lawton, Safaee, Burkhardt. Approved the final version of the manuscript on behalf of all authors: Lawton. Statistical analysis: Kamide. Administrative/technical/material support: Lawton. Study supervision: Lawton.

\section{Correspondence}

Michael T. Lawton: Barrow Neurological Institute, Phoenix, AZ. michael.lawton@barrowbrainandspine.com. 